\title{
Preliminary Report: Another Perspective on the Effect of Prolonged Stretching for Joint Contractures
}

J. Phys. Ther. Sci.

19: 97-101, 2007

\author{
TAKeYa Ono, PhD, RPT ${ }^{1)}$, Mariko Tsubol, RPT ${ }^{2}$, SADAAKi OKI, PhD, MD ${ }^{1)}$, \\ Michele EISEMANN SHIMIZU, PhD, RPT ${ }^{1)}$, AKIRA OTSUKA, PhD, RPT ${ }^{1)}$, \\ KAYOKO SHIRAIWA, PhD, RPT ${ }^{1)}$, WATARO MATUSHITA, PhD, MD ${ }^{3)}$, \\ HIROKI KAJIHARA, PhD, $\mathrm{MD}^{1)}$ \\ 1)Department of Physical Therapy, Faculty of Health Sciences, Prefectural University of \\ Hiroshima: 1-1 Gakuen Machi, Mihara City, Hiroshima, Japan, 723-0053, \\ TEL +81 848-60-1210,FAX +81848-60-1226,E-mail: ono@pu-hiroshima.ac.jp \\ 2) Department of Physical Therapy, Yamamoto Orthopaedic Clinic \\ ${ }^{3)}$ Matushita Orthopaedic Clinic
}

\begin{abstract}
The purpose of this study was to re-examine the influence of prolonged stretching on joint contractures by using a specific force for joint correction and ROM measurement. Twenty-five 8-week-old female Wistar rats were divided into five groups. One group was used as a control (G1), and the rats in the four experimental groups were immobilized to cause ankle contractures. The first group was immobilized continuously (G2). In the second, third and fourth groups, the casts were removed daily, and the foot was held in dorsiflexion for periods of 30 minutes (G3), 60 minutes (G4), and 120 minutes (G5), respectively. After a week, restriction in ROM in G4 and G5 significantly increased, as compared to G2 and G3, and contraction bands were observed in G4 and G5. These results suggest that aggravation of ROM restriction increased with the amount of stretching, and the cause may be a decrease in muscle circulation.
\end{abstract}

Key words: Joint contracture, Prolonged stretching, Contraction band

(This article was submitted Sep. 11, 2006, and was accepted Oct. 27, 2006)

\section{INTRODUCTION}

Restriction of joint range of motion (ROM) is a common clinical problem found in patients receiving physical therapy. It has been reported in animal experiments that joint contractures can be prevented by prolonged stretching for about 30 minutes daily ${ }^{1,2)}$. In these experiments, ROM was measured manually with a goniometer, but the amount of pressure used to measure the ROM was not reported. Also, in these experiments, adhesive tape was used to hold the ankle joint in position for the prolonged stretching, but how much pressure was used for the joint correction was not indicated.
Joint excursion can increase due to an increase in the amount of the force used in the joint correction ${ }^{3,4)}$; therefore, to understand joint excursion, the relationship between joint correction power and joint excursion must be taken into consideration ${ }^{3,4)}$. The purpose of this study was to re-examine the influence of prolonged stretching on joint contractures with a specific force used to measure and to correct joint ROM.

Regarding the amount of force necessary to correct restriction of human ankle joint dorsiflexion, Kottke $\mathrm{FJ}^{5)}$ stated that it is necessary to use about an average of $9 \mathrm{~kg}(4.5-13.5 \mathrm{~kg})$. The average weight of Japanese men in the 40-year age 
bracket is about $65 \mathrm{~kg}^{6}$ ), and $9 \mathrm{~kg}$ is equivalent to about $15 \%$ of that weight. The average weight of a female Wister rat is $200 \mathrm{~g}$ at eight weeks of age, and $15 \%$ of that weight is about $30 \mathrm{~g}$. Therefore, in this study, a $30 \mathrm{~g}$ force was used to measure and correct the ankle joint movements of rats.

\section{MATERIALS AND METHODS}

\section{Rats}

Twenty-five 8-week-old female Wistar rats with a controlled initial body weight of $190 \mathrm{~g}$ to $200 \mathrm{~g}$ were used. The experiments were conducted in accordance with our university's Guidelines for Animal Experimentation and the U.S. National Institute of Health guidelines. The animals were housed in a temperature-controlled room at $20^{\circ} \mathrm{C}$ with a 12-hour light-dark cycle. The rats were provided free access to standard rat food and to water.

\section{Procedures to immobilize and stretch the ankles}

As a control, five rats were used (G1). Twenty rats out of the 25 were anesthetized with Nembutal $(0.5 \mathrm{~mL} / \mathrm{kg})$, and their right ankle joints were fixed in full plantar flexion for one week by plaster casts, the soleus muscle being immobilized in the shortened position. These twenty rats were divided into four groups as follows. The first group was immobilized continuously (G2). The casts of the second, third and fourth groups were removed daily, and the right foot was held in dorsiflexion by a spring balancer set at a force of $30 \mathrm{~g}$, for periods of 30 minutes (G3), 60 minutes (G4), and 120 minutes (G5), respectively (Fig. 1). If the ROM changed as the ankle stretched, the rat was repositioned so that the spring balancer would always apply a force of $30 \mathrm{~g}$. After the stretching session was completed, the casts were then reapplied, again with the ankle fixed in full plantar flexion. The control group was anesthetized with Nembutal anesthesia $(0.5 \mathrm{~mL} / \mathrm{kg})$ every day, the same as the other groups.

\section{Measurement of ROM of ankle joint dorsiflexion}

Before the experiment and after one week, all the rats were anesthetized with Nembutal $(0.5 \mathrm{~mL} / \mathrm{kg})$. The ROM of ankle joint dorsiflexion was measured as follows. First, the rat was positioned in sidelying. To measure dorsiflexion, a force of $30 \mathrm{~g}$ was applied vertically to the sole of the foot by a tension

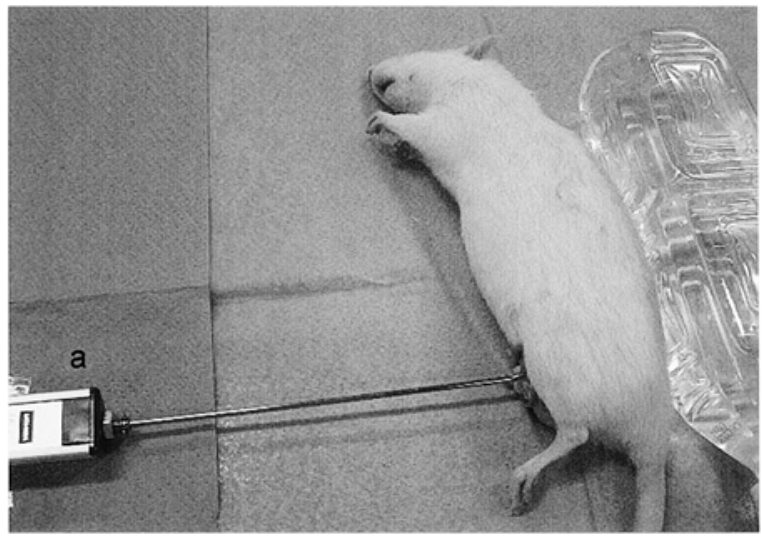

Fig. 1. Daily prolonged stretching by a spring balancer under Nembutal. A force of $30 \mathrm{~g}$ was applied to the hind limb to obtain dorsiflexion. a: Spring balancer.

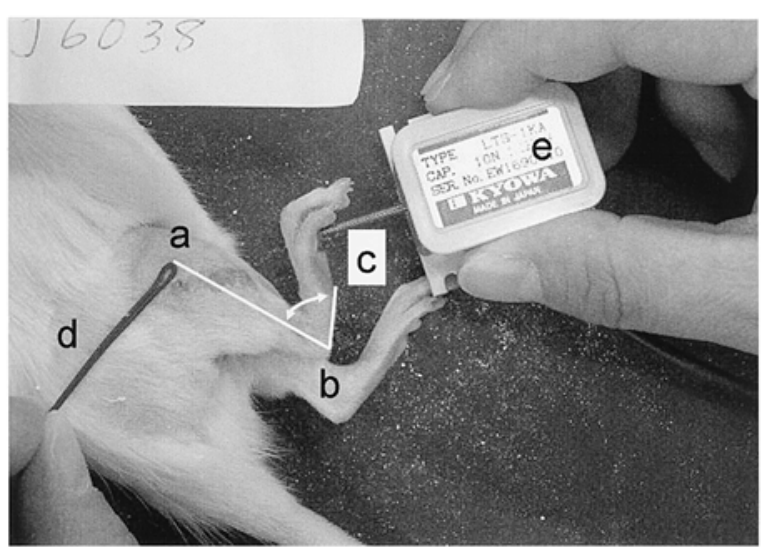

Fig. 2. Ankle ROM measurement using a tension meter. Angle of dorsiflexion was defined as angle a-b-c. Tip of black stick indicates the head of the fibula. $a-b$ : parallel line from the head of the fibula to the bottom of the heel. b-c: parallel line along the bottom of the heel. d: black stick. e: tension meter.

meter (KYOWA Co, LTS-1KA) (Fig. 2). Then, a digital photograph was taken from directly above the hind limb, and the angle of dorsiflexion was measured on a computer (Adobe Photoshop 6.0). The ROM of dorsiflexion was defined as the angle obtained from a line parallel to the longitudinal axis of the fibula and a line parallel to the bottom of the heel, to eliminate forefoot movement from the measurement. When the ROM was measured, the knee joint was flexed. 
Morphological analysis of the soleus muscles

After sacrificing the rats with Nembutal $(5 \mathrm{~mL} /$ $\mathrm{kg}$ ), one of the legs in each group was isolated and cut at the knee joint. The ankle joint was fixed by pinning as follows: a wire was introduced into the tibia through the fully plantar flexed ankle joint to the calcaneus to maintain the soleus in the shortened positioned. The leg was then fixed in a $10 \%$ formalin solution and embedded in paraffin. After one week of formalin fixation, the soleus muscles were stained with hematoxylin-eosin for histologic examination.

\section{Statistics}

The Kruskal-Wallis test was used to compare the changes in ROM dorsiflexion before and after the experiments in each group. Fisher's PLSD post hoc test was used to identify significant comparisons. The accepted alpha level of all of the statistical processing was at the 0.05 level.

\section{RESULTS}

The changes in ROM are shown in Table 1. Statistically, compared to G1, ROMs in G2, G3, G4, and G5 were significantly decreased. ROM decrease in G2 was no different than G3, but the decreases in ROM were significantly greater in G4 and G5; however, ROM decrease in G4 was not significantly different from G5. According to these results, no positive effects could be seen for prolonged stretching of joint contractures.

There were no morphological changes noted in the soleus muscles of $\mathrm{G} 2$ and G3; however contraction bands ${ }^{7,8)}$ were seen in G4 and G5. In addition, more contraction bands were seen in G5 than in G4 (Fig. 3).

\section{DISCUSSION}

The purpose of our study was to re-examine the influence of prolonged stretching on joint contractures, as researched by Williams ${ }^{1)}$ and Nakata et $\mathrm{al}^{2}$ ). Our results indicate that 30 minutes of prolonged stretching (G3) does not prevent the development of joint contractures, as can be seen in the comparison of $\mathrm{G} 2$ and $\mathrm{G} 3$, where no difference was found between the two groups. Also, when compared with $\mathrm{G} 2$, it can be seen that prolonged stretching for 60 minutes and 120 minutes, as in G4 and G5, resulted in significantly increased joint contractures. These results differ from previous reports, which recommended about 30 minutes of stretching to prevent joint contracture development.

Two different methods were used in previous research experiments and in ours. One was a different method of ROM measurement. Previous researchers manually measured ROM with a goniometer, but we believe that to accurately measure joint ROM a tension meter is necessary ${ }^{9}$. Therefore, we used a tension meter to avoid the subjective aspect of ROM measurement. The other difference was the method of prolonged stretching. Previous researchers used a single application of adhesive tape to achieve the prolonged stretching of ankle dorsiflexion, which we believe does not adequately apply a steady force at the joint if the ROM gradually changes during prolonged

Table 1. The changes in ankle dorsiflexion ROM (degrees)

\begin{tabular}{cccccc}
\hline & G1 $^{\text {bcde }}$ & G2 $^{\text {ade }}$ & G3 $^{\text {ade }}$ & G4 $^{\text {abc }}$ & G5 $^{\text {abc }}$ \\
\hline 1 & 37 & 70 & 68 & 87 & 87 \\
2 & 39 & 60 & 65 & 90 & 89 \\
3 & 38 & 65 & 73 & 68 & 90 \\
4 & 41 & 70 & 69 & 70 & 77 \\
5 & 40 & 70 & 76 & 95 & 78 \\
\hline Mean & 39 & 67 & 70 & 82 & 84 \\
SD & 1 & 4 & 4 & 12 & 6
\end{tabular}

G1: control, G2: immobilized one week continuously, G3: 30 minutes dorsiflexion daily, G4: 60 minutes dorsiflexion daily, G5: 120 minutes dorsiflexion daily.

a: $p<0.05$ (compared with G1), b: $p<0.05$ (compared with G2), c: $\mathrm{p}<0.05$ (compared with G3), d: $\mathrm{p}<0.05$ (compared with G4), e: $\mathrm{p}<0.05$ (compared with G5) . 

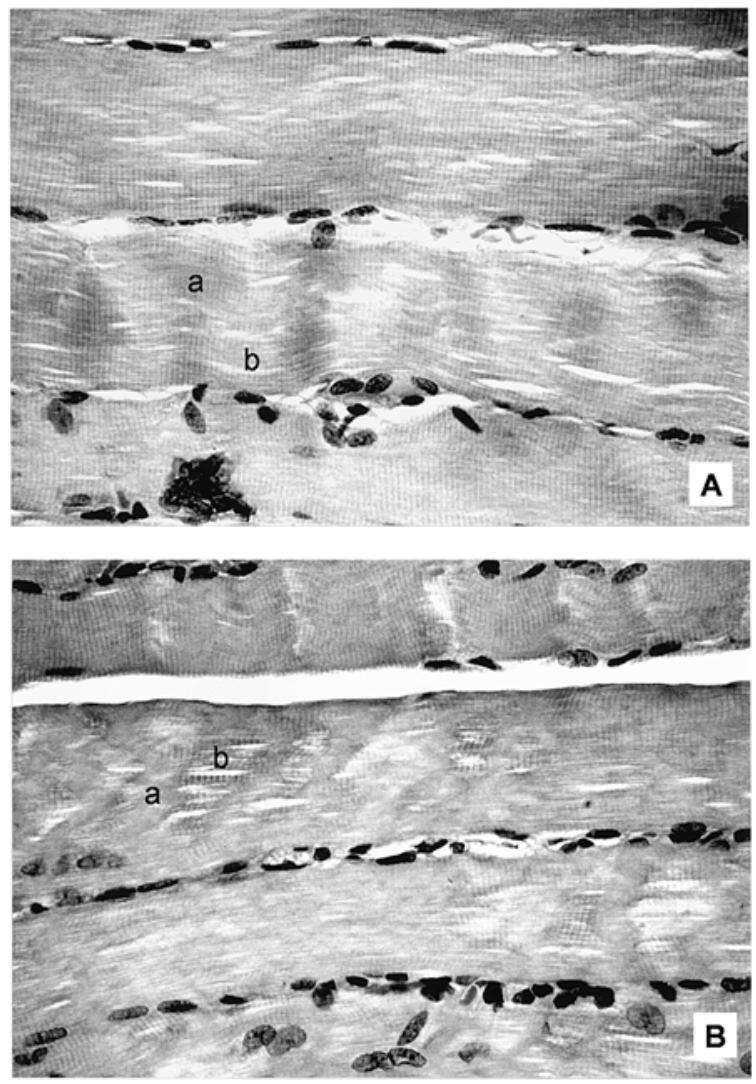

Fig. 3. Contraction band (HE stain, original magnification $\times 400$ ). Longitudinal section of soleus muscle in G4 and G5. Sites marked with a and b indicate one example of the contraction band, although other sites can be seen along the fiber. A: G4moderate contraction band, B: G5- severe contraction band, a: Site of contraction, b: Site of elongation.

stretching. It has been reported that stretching should be done at the terminal range of motion to be effective $^{4)}$. For this reason, clinically, stretching is done by using a pulley system with weights to always adjust the stretching force to a change in the terminal $\mathrm{ROM}^{5)}$, and our method of joint contracture stretching with a spring balancer is a recreation of the clinically used pulley system. The difference in the results of our experiments and those of the previous researchers may be the difference in the experimental methods.

Our results indicate that ROM was aggravated by an increased stretch time. During prolonged stretching of the ankle joint, the soleus muscle was stretched. As the period of stretching increased, elongation of muscle fibers during the stretching most probably reduced muscle circulation ${ }^{10,11)}$. Alterations in the muscles appeared, which seem to depend on the periods of decreasing muscle circulation $^{12)}$. In fact, contraction bands were found in the soleus muscles of G4 and G5, more so in G5. It seems that the contraction bands caused an increased ROM restriction in the ankle joint. The cause of the contraction band has been attributed to reperfusion injury after ischemia ${ }^{7}, 8$ ), and the histological changes of the contraction band indicated partial contraction of serial muscle fibers with actin and myosin ${ }^{13)}$. We think that the contraction band caused the muscle to harden, due to the contraction of actin and myosin, but our findings were obtained with only a few specimens. Other causes of ROM restriction (i.e. skin, joint capsule, cartilage) need to be taken into consideration. Further work is currently underway to determine whether prolonged stretching is effective or not for the prevention of joint contracture using more cases and further quantitative measurements.

\section{REFERENCES}

1) Williams PE: Use of intermittent stretch in the prevention of serial sarcomere loss in immobilised muscle. Ann Rheum Dis, 1990, 49: 316-317.

2) Nakata A, Okita M, Nakai $K$, et al.: Effects of prolonged stretching for prevention of joint contracture in mice. Rigaku Ryohogaku, 2002, 29: 1-5 (in Japanese).

3) Flowers KR, Pheasant SD: The use of torque angle curves in the assessment of digital joint stiffness. J Hand Ther, 1988, 2: 69-74.

4) Flowers KR, LaStayo P: Effect of total end range time on improving passive range of motion. J Hand Ther, 1994, 7: 150-157.

5) Kottke FJ, Pauley DL, Ptak RA: The rationale for prolonged stretching for correction of shortening of connective tissue. Arch Phys Med Rehabil, 1966, 47: 345-352.

6) Isokawa M, Imanaka K, Ootuki F, et al.: New standard value of Japanese physical strength. Tokyo: Fumaido Publishing, 2000, pp 70-77 (in Japanese).

7) Miyazaki S, Fujiwara H, Onodera $T$, et al.: Quantitative analysis of contraction band and coagulation necrosis after ischemia and reperfusion in the porcine heart. Circulation, 1987, 75: 1074-1082.

8) Kajihara H: Pathology of acute myocardial infraction: with special reference to morphological changes of myocardial cells and repair of infarcted area. Cardioangiology, 1986, 26: 49-58 (in Japanese).

9) Ono $\mathrm{T}$, Aoyama H, Komazawa H, et al.: Comparison 
of two methods of goniometry. Yamagata Journal of Health Science, 2000, 3: 55-57 (in Japanese).

10) Yokozawa H, Muraoka $Y$, Shimizu S, et al.: Changes in fascicle length and muscle oxygenation during passive static stretching. The Journal of Japanese College of Angiology, 2002, 42: 25-28 (in Japanese).

11) Hara T, Takuma $Y$ : Effect of prolonged stretching of the gastrocnemius on local muscle oxygenation.
Rehabilitation Journal of Tosa, 2004, 3: 31-35 (in Japanese).

12) Appell HJ, Gloser S, Duarte JA, et al.: Skeletal muscle damage during tourniquet-induced ischaemia. The initial step towards atrophy after orthopaedic surgery? Eur J Appl Physiol Occup Physiol, 1993, 67: 342-347.

13) Sugita H, Ozawa E, Nonaka I: Principles of myology. Tokyo: Nankodo, 1995, pp 366-374 (in Japanese). 